to which the above-mentioned investigations lead are represented on the Fig. 2 (isotopes with zero nuclear moment): the lengths of the respective lines indicate their relative intensities. These intensities, or the absorption coefficients, do not give us directly the proportion of the various kinds of isotopes, because the probability of the transitions may not be the same for different isotopes, which was demonstrated by me in the case of the 2537 A. line. ${ }^{2}$ For this reason a correlation based on the intensities, as Schüler and Keyston have done (cadmium, thallium), cannot be considered as convincing.

In the case of the line $2537 \mathrm{~A}$., calculations can be made using the isotope concentrations given by Aston, which are not subjeet to the above-mentioned objections, for the life period of different components is known.2 They show that if we attribute the lines 0 and $+11.5 \mathrm{~mA}$. to the isotopes 200 and 202 (in arbitrary succession), the three lines represented on Fig. 1 must be attributed to two isotopes, 199 and $20 \mathrm{I}$ (overlapping of a doublet and a triplet ?), with nuclear moments equal to an odd multiple of $1 / 2$. This last conclusion follows from the fact that in the parallel Zeeman effect the parallel zero components, which show a Paschen-Back effect in higher magnetic fields, are not observed.

It is curious that the direction of the isotope shift of the lines shown in Fig. 2 is opposite to that observed with thallium and to that resulting from the motion of the nucleus around the centre of gravity of the atom. This shift has at the same time a relatively high value of $c .0 \cdot 2 \mathrm{~cm} .^{-1}$ for each addition of two protons, which is, however, nearly a half of the shift observed on the line 5351 A. of thallium. ${ }^{x}$ It is produced probably by the modification of the electric field surrounding the nucleus, as resulting from the addition or subtraction of two protons.

A full report of these investigations will be published shortly in the Bull. Acad. Polonaise. S. Mrozowski.

Physical Laboratory of the Society of Sciences and Letters, Warsaw, May 5.

\footnotetext{
${ }_{1} \mathrm{H}$. Schüler and J. E. Keyston, Die Naturwissenschaften, 19, p, 320 ; 1931.

S. Mrozowski, Bull. Acad. Pol., p. 464; 1930 ; in part also N Fiture, 126, p. $684 ; 1930$

S. Mrozowski, Phys, Review, 37, p. $845 ; 1931$

- S. Mrozowski, Zeit. für Physik, 68, p. 278; 1931.
}

\section{Electron Polarisation.}

IF the free electron has the spin theoretically assigned to it, the theory ${ }^{1}$ of the double scattering of electrons by single stripped nuclei would lead one to expect that when an electron beam impinged successively on two metal targets, the intensity of the secondary seattering would be asymmetric about the direction af incidence of the secondary beam.

The majority of the previous experiments to detect this effect have been carried out either with slow electrons (a few hundred volts energy), small scattering angles, or reflectors of low atomic number. Under these conditions no observable asymmetry is predicted by theory, and this is in accord with the results of most investigators.

Chase ${ }^{2}$ has reported that with $90^{\circ}$ scattering from $45^{\circ}$ lead targets, and with primary electron velocities of from 0.7 to 0.95 the velocity of light, the effect does appear, together with more striking features not predicted by theory. With these high velocities, how. ever, the theory loses much of its meaning, since in its formulation the radiative forces on the electron were neglected. It seemed desirable, therefore, to look for the effect with a primary electron velocity

$$
\text { No. 3215, VoL. 127] }
$$

of such a value that the predicted asymmetry should be easily observable, and yet the radiative forces should be small.

The tube was constructed so that the electron beam reflected at $90^{\circ}$ from a $45^{\circ}$ tungsten polarising target impinged at normal incidence on a tungsten analysing target. The intensities of the secondary scattering at an angle of slightly more than $90^{\circ}$ could be measured at $0^{\circ}$ and $180^{\circ}$ of the azimuth without altering any part of the apparatus. Only the faster group of electrons in the scattered beams was allowed to reach the measuring instrument, all others being prevented by the use of suitable retarding fields (1700 volts for a primary voltage of $10 \mathrm{kv}$.). Rotation of the polarising target through $180^{\circ}$ about the direction of the secondary beam, and the use of a second filament at $180^{\circ}$ to the first, was intended to reverse the spin asymmetry and to provide a check against possible diffraction effects in the analyser.

It was found that with $10 \mathrm{kv}$. and $1 \mathrm{kv}$. electrons the ratio of the intensities at $0^{\circ}$ and $180^{\circ}$ of the azimuth did not differ by more than the experimental error ( 1 per cent). For purely nuclear, ideal scattering (in the sense of the Mott theory) the difference should have been approximately 13 per cent. This figure is based on the theoretical idea that the probability of an electron being scattered in a given direction depends on the orientation of its spin axis. It would therefore attain this value only if all electrons scattered 'into' the polarising target were rediffused with appreciable energy loss, so that the faster group of electrons in the secondary beam would be polarised to the degree predicted by theory.

It would appear to follow from this experiment that either the free electron has not the spin theoretically assigned to it, or that the present approximate theory with its neglect of orbital electron scattering and radi. ative forces predicts an effect which would not appear in a more complete formulation.

I am indebted to Prof. O, W, Richardson for the suggestion of the problem.

King's College,

Strand, London, W.C.2, May 8.

1 Mott, Proc. Roy. Soc., A, 124, $425 ; 1929$.
2 Pht. Rev., 36, 1060 ; 1930.

Hyperfine Structure in the Spectrum of Copper.

T'He principal copper doublet at $\lambda \lambda 3248$ and 3275 has been studied with great care in the fourth and fifth orders of the large concave grating of the Physical Institute at Tübingen. Each line consists of a very sharp, narrow doublet of separation, $0.043 \pm 0.001 \mathrm{~A}$. or $0.41 \pm 0.01 \mathrm{~cm} .^{-1}$, the long wave-length component being about twice as strong as the short wave-length component.

Extreme care must be exercised in the photography of this pair of lines. They are the "raies ultimes' of copper, and even when the copper content of the electrodes is so low as 0.001 per cent, the fine structure of the lines indicates the beginning of self-reversal. A similar phenomenon was noticed by Royds ${ }^{1}$ in the green line of thallium.

So far as we are aware, the only previous work on the fine structure of these two lines was done by Back ${ }^{2}$ with the same apparatus as that used by us. On the basis of the present work, it seems that Back's results can be explained by self-reversal of the fine-structure components. It was possible, with 0.001 per cent copper in the electrode and the use of high excitation, to reproduce the results of Back. Only when the copper content of the electrode had been reduced to 